
\title{
3 Research Square \\ Local Recurrence in Young Women with Breast Cancer: Breast Conserving Therapy vs. Mastectomy Alone
}

\section{Dang Van Nguyen}

Hanoi Medical University

\section{Sang-Won Kim}

Konyang University Hospital

Young-Taek Oh

Ajou University Hospital

O Kyu Noh

Ajou University Hospital

Yongsik Jung

Ajou University Hospital

Dae Sung Yoon

Konyang University Hospital

Mison Chun ( $\nabla$ chunm@ajou.ac.kr)

Ajou University Hospital

\section{Research Article}

Keywords: breast cancer, breast conservation therapy, total mastectomy, young women, local recurrence

Posted Date: February 19th, 2021

DOl: https://doi.org/10.21203/rs.3.rs-209244/v1

License: (c) (i) This work is licensed under a Creative Commons Attribution 4.0 International License. Read Full License 


\section{Abstract}

We compared cumulative incidence of local recurrence in young patients ( $\leq 40$ years) with breast cancer between breast conserving therapy (BCT) and mastectomy alone. Among 428 women with early-stage breast cancer treated between 2001 and 2012, 311 underwent BCT and 117 underwent mastectomy alone. Adjuvant systemic treatments were administered to 409 patients (95.6\%). We compared the cumulative incidence of LR and survival rates between two groups. During a median follow-up period of 91 months, the 10-year cumulative incidence of LR was 9.3\% (median interval of 36.5 months from surgery). Patients treated with BCT tended to have a higher risk for local recurrence $(11.1 \%$ for BCT vs. $4.1 \%$ for mastectomy alone, $\mathrm{p}=0.078)$. All patients with isolated LR after BCT $(n=23)$ underwent salvage mastectomy followed by systemic treatments. The 5-year distant metastasis-free survival and overall survival of patients with isolated LR after BCT were $44.2 \%$ and $82.2 \%$, respectively. The BCT group exhibited approximately a 2.5 -fold higher risk of LR than mastectomy alone group. Patients with isolated LR after BCT showed poor prognosis despite undergoing aggressive salvage treatments. More effective long-term follow-up strategies should be established to reduce LR for improving prognosis and preserving cosmetic outcomes in young women.

\section{Introduction}

Breast conservation therapy (BCT) is the preferred primary local treatment for early-stage, invasive breast cancer because of its equivalent survival to that of mastectomy, with the additional advantage of preserving cosmetic outcomes of the involved breast.

However, performing BCT in young patients has remained challenging. As young age has been reported to be an independent risk factor for high local recurrence and poor prognosis ${ }^{1-3}$, more aggressive local treatment such as mastectomy was often considered for young patients. In addition, including a small number of young patients in early randomized trials made it difficult to confirm the efficacy and safety of BCT in young women 4,5 .

Previous studies have been published to address unconfirmed legitimacy of BCT for young patients ${ }^{6-20}$, and consistently demonstrated similar survival between BCT and mastectomy. However, the majority of these were retrospective studies and it could not draw a definite conclusion. Several studies had problematic study designs, including patients who received postmastectomy radiation therapy ${ }^{7-12}$, $14,16,19,20$. In addition, only a few studies have analyzed treatment outcomes in recently treated patients $16,18,20$

As preservation of cosmetic outcomes of breast is an important goal of BCT, local control should be seriously concerned before determining definitive local treatment modality. However, comparison of local control between BCT and mastectomy has been of little interest because of similar survival. It has been questioned whether BCT also shows comparable local control with that of mastectomy because several studies have demonstrated the superiority of mastectomy in terms of local control 6,10,12,15,19,20. 
Diagnosis with recurrence comes psychologically as a disaster for patients even though its extent is limited and can be salvaged by mastectomy. Furthermore, as repeated hospitalization, surgery, and adjuvant treatments are undertaken, additional medical costs and longer treatment duration can create burdens for patients.

In this study, we compared the incidence of local recurrence (LR) in young women with breast cancer between BCT and mastectomy alone. In addition, we investigated risk factors associated with increased LR among patients who underwent BCT.

\section{Results}

\section{Patient characteristics}

We identified 428 patients who met our study criteria. Of these, 311 patients (72.7\%) underwent BCT and 117 (27.3\%) underwent total mastectomy alone. A comparison of patient characteristics between both treatment groups is summarized in Table 1. The median age of entire patients was 37 years (range, 1940 years). The median tumor size was similar between both treatment groups ( $1.7 \mathrm{~cm}$ for the BCT group vs. $2.0 \mathrm{~cm}$ for the mastectomy alone group). Among pathological factors, human epidermal growth factor receptor 2 status and the number of positive lymph nodes were significantly different between two treatment groups. 
Table 1

Patient characteristics

\begin{tabular}{|c|c|c|c|}
\hline Variables & BCT $(n=311)$ & Mastectomy $(n=117)$ & $p$ value \\
\hline Age at diagnosis (years) & 36 & 37 & 0.041 \\
\hline \multicolumn{4}{|l|}{ Median } \\
\hline Histologic grade & $136(46.7 \%)$ & $53(50.5 \%)$ & \multirow[t]{3}{*}{0.587} \\
\hline Low to intermediate & $155(53.3 \%)$ & $52(49.5 \%)$ & \\
\hline \multicolumn{3}{|l|}{ High } & \\
\hline Estrogen receptor & $104(33.4 \%)$ & $31(26.7 \%)$ & \multirow[t]{3}{*}{0.226} \\
\hline Negative & $207(66.6 \%)$ & $85(73.3 \%)$ & \\
\hline \multicolumn{3}{|l|}{ Positive } & \\
\hline Progesterone receptor & $100(32.2 \%)$ & $36(31.0 \%)$ & \multirow[t]{3}{*}{0.917} \\
\hline Negative & $211(67.8 \%)$ & $80(69.0 \%)$ & \\
\hline \multicolumn{3}{|l|}{ Positive } & \\
\hline HER2 & $258(83.2 \%)$ & $71(61.7 \%)$ & \multirow[t]{3}{*}{$<0.001$} \\
\hline Negative & $52(16.8 \%)$ & $44(38.3 \%)$ & \\
\hline \multicolumn{3}{|l|}{ Positive } & \\
\hline Tumor size & 207 (66.6\%) & $68(58.1 \%)$ & \multirow[t]{3}{*}{0.131} \\
\hline$\leq 2 \mathrm{~cm}$ & $104(33.4 \%)$ & $49(41.9 \%)$ & \\
\hline \multicolumn{3}{|l|}{$>2 \mathrm{~cm}$} & \\
\hline No. of positive LNs & $247(79.4 \%)$ & $74(63.2 \%)$ & \multirow[t]{3}{*}{0.001} \\
\hline 0 & $64(20.6 \%)$ & $43(36.8 \%)$ & \\
\hline \multicolumn{3}{|l|}{$1-3$} & \\
\hline Adjuvant chemotherapy & $75(24.1 \%)$ & $30(25.6 \%)$ & \multirow[t]{3}{*}{0.841} \\
\hline No & $236(75.9 \%)$ & $87(74.4 \%)$ & \\
\hline \multicolumn{3}{|l|}{ Yes } & \\
\hline Adjuvant endocrine therapy & $92(29.6 \%)$ & $35(29.9 \%)$ & \multirow[t]{3}{*}{0.791} \\
\hline No & 219 (70.4\%) & $82(70.1 \%)$ & \\
\hline Yes & & & \\
\hline $\begin{array}{l}B C T \text {, breast conserving thera } \\
\text { node; }\end{array}$ & human epide & wth factor receptor ty & ymph \\
\hline
\end{tabular}




\section{Local recurrence}

The median follow-up period was 91 months for all patients (range, 8-192 months. There were 30 patients (26 patients in the BCT group and 4 in the total mastectomy alone group) who experienced LR as the first recurrence, with a median interval of 36.5 months between initial local treatment and the date of pathologic confirmation. Of these, 25 patients developed isolated LR ( 23 patients in the BCT group and 2 patients in the total mastectomy alone group). All patients with isolated LR underwent salvage total mastectomy (BCT group) or wide excision (total mastectomy alone group).

The 10-year cumulative incidence of LR was $9.3 \%$ for all patients. Patients who underwent BCT showed a tendency toward higher cumulative incidence of LR, compared with those in the total mastectomy alone group ( $11.1 \%$ in the BCT group vs. $4.1 \%$ in the total mastectomy alone group, $p=0.078$ ) (Fig. 1). Multivariate competing risk regression analysis showed that BCT had a tendency toward increased risk of LR (relative risk, 3.182; 95\% confidential interval, $0.0921-1.27 ; p=0.064)$. Other factors including histologic grade $(p=0.63)$, estrogen receptor $(p=0.80)$, progesterone receptor $(p=0.54)$, HER2 $(p=0.18)$ and tumor size $(p=0.31)$ were not found to be significantly associated with increased risk of LR.

We investigated to identify factors for increased risk of LR in the BCT group. On univariate analysis, histologic grade, hormone receptor status and tumor size were not significantly associated with increased the risk of $\operatorname{LR}$ (all $p>0.1$ ).

\section{Survival rates after local recurrence}

Patients with LR showed significantly worse estimated 10-year DMFS than those without LR (47.0\% vs. $88.0 \% ; p<0.001)$. For 23 patients with isolated LR after BCT, 7 patients experienced distant metastasis and the estimated 10-year DMFS rate after LR was $44.2 \%$ with a median period of 54 months (Fig. 2).

The estimated 10 -year OS rate was $89.0 \%$ for all patients. Patients with LR showed significantly worse estimated 10-year OS than those without LR $(66.5 \%$ vs. $91.1 \% ; p<0.001)$. For patients with isolated LR after BCT, the estimated 10-year OS rate was $51.4 \%$ (Fig. 3).

\section{Discussion}

Previously, the notion that breast cancer arising in young age presents more aggressive pathological features with advanced stage and young age is associated with increased risk of recurrence acted as barriers to perform BCT in young patients ${ }^{1-3}$. However, this concern may be unwarranted as previous studies have reported consistently equivalent survival between BCT and mastectomy ${ }^{6-20}$. The treatment outcomes of young patients undergoing BCT have also improved, owing to better preoperative imaging work-ups and advances in adjuvant treatments ${ }^{16,21,22}$. Based on this evidence, BCT is currently recommended as the first option whenever suitable, even in young patients ${ }^{23,24}$. 
Despite the evidence showing equivalent survival between BCT and mastectomy, young patients with breast cancer in the United States are increasingly choosing mastectomy instead of BCT ${ }^{25,26}$. Several factors may influence this phenomenon, including living conditions that make it difficult to receive conventional radiation therapy and fear of higher risk of LR, leading to subsequent repeated surgery ${ }^{17}$.

Patients' fears are not vague because several studies have reported higher rates of $L R$ in young patients treated with BCT 6,10,12,15,19,20. In this study, patients in the BCT group had a 2.5-fold higher risk of LR than those in the total mastectomy alone group and all 23 patients with isolated LR after BCT eventually underwent salvage mastectomy. The higher cumulative incidence of LR in the BCT group is supported by a recent prospectively observational cohort study (10-year LR rates of $11.7 \%$ in the BCT group vs. $4.9 \%$ in the mastectomy group, $p<0.001)^{20}$.

Interestingly, the cumulative incidence curve in this study indicates that the risk of LR in the BCT group increased constantly over time, whereas a plateau was reached after 6 years in the total mastectomy alone group. van der Sangen et al also reported similar patterns of LR according to the primary local treatments in young women ${ }^{10}$. Due to the continuous increase of LR in the BCT group, the difference in the incidence of LR between BCT and total mastectomy alone group will increase over time.

In this study, approximately one-third of patients with isolated LR after BCT suffered from the development of distant metastasis, even though they underwent aggressive salvage treatments. Anderson et al. also reported that of 342 patients with isolated LR after BCT, 127 (37.1\%) experienced distant metastasis ${ }^{27}$. Given the poor DMFS after aggressive salvage treatments for isolated LR in the BCT group ( $44.2 \%$ at 5 years), more effective novel systemic treatments should be investigated. More importantly, follow-up strategies should be improved to find suspected benign lesions with the potential to become malignant because risk factors associated with increased risk of LR after BCT were not identified.

Constantly increased incidence of LR and secondary development of distant metastasis in the BCT group can be dealt with by identifying patients with risk factors for LR and monitoring them intensively. Therefore, we investigated risk factors associated with increased risk of LR in the BCT group. However, no clinical or pathological factors were significantly associated with increased LR. Previously, a few researchers investigated to identify risk factors associated with LR after BCT and they did not find any relevant factors ${ }^{10,21,22}$. This suggests that decisions for definitive local treatment approaches should not be solely based on clinical and/or pathological factors in young women. Further studies are warranted to examine risk factors at the molecular level.

This study had several limitations including inherent biases due to its retrospective design. The local treatment approach was determined according to the surgeons' discretion or patients' preference. Therefore, the distribution of some factors was not balanced. Although we adjusted for all available clinical and pathological factors, other unknown confounders might influence treatment outcomes. In 
addition, this study analyzes data from two institutions and it is difficult to generalize the results. However, all the details of local and systemic treatments were performed based on standard procedures.

In conclusion, patients in the BCT group exhibited approximately a 2.5-fold increased risk of LR compared with those in the mastectomy alone group. The incidence of LR increases continuously in the BCT group in contrast to the mastectomy alone group. Furthermore, one-third of patients with an isolated LR after BCT experienced distant metastasis despite of aggressive salvage mastectomy followed by systemic treatments. Although BCT had equivalent OS to total mastectomy alone and it can be recognized as the first local treatment option for young women with breast cancer, countermeasures are required to improve quality of life in patients treated with BCT through preservation of breast cosmetic outcomes. To reduce the risk of distant metastasis and to improve prognosis in patients with isolated LR after BCT, more effective systemic treatments should be investigated. Ultimately, it is more important to reduce the incidence of LR by establishing thorough long-term follow-up strategies for improved prognosis, as well as preserving cosmetic outcomes in young women undergoing BCT.

\section{Materials And Methods}

The Institutional Review Board of two participating institutions (Konyang University Hospital Institutional Review Board and Ajou Institutional Review Board) approved this study with a waiver of informed consent due to its retrospective nature. All procedures performed were in accordance with the ethical standards of the institution and with the 1964 Helsinki declaration (and its later amendments).

We reviewed the medical records of all patients with breast cancer aged $\leq 40$ years at diagnosis who were curatively treated between 2001 and 2012. Patients diagnosed with inflammatory breast cancer, ductal carcinoma in situ, or malignancies other than carcinoma were excluded from the analysis, as were those treated with neoadjuvant therapy or postmastectomy radiation therapy and those who refused radiation therapy after partial mastectomy.

All patients underwent definitive local treatment with either BCT or total mastectomy alone. If patients underwent total mastectomy subsequently after partial mastectomy because of a positive resection margin, local treatment was defined as mastectomy. Local treatment approaches were determined according to the surgeons' discretion or patients' preference. Axillary evaluation was performed with sentinel lymph node biopsy or limited axillary dissection.

Adjuvant chemotherapy was administered in 323 patients (75.5\%). For patients with negative lymph nodes, the chemotherapeutic drug was either a combination of cyclophosphamide, methotrexate, and 5fluorouracil, or an anthracycline-based regimen. Patients with positive lymph nodes received a taxanebased regimen. Adjuvant hormone therapy was administered to all patients with positive hormone receptor status. In total, 406 patients $(95.6 \%)$ received adjuvant systemic treatments.

Patients treated with BCT received whole-breast irradiation of 45-50 Gy with a conventional fractionation scheme, followed by a boost to the tumor bed with a median dose of $14 \mathrm{~Gy}$. For patients with multiple 
lymph nodes, radiation was delivered conventionally to the supraclavicular area with a total dose of 4550 Gy.

Categorical variables of clinico-pathological factors were compared between both treatment groups using the Chi-squared or Fisher's exact test. Continuous variables, such as age and primary tumor size, were compared using the Kruskal-Wallis test. We assessed LR using cumulative incidence analysis (Gray's test). LR was defined as the first recurrence occurring at the ipsilateral breast or chest wall. Competing risks included regional recurrence, distant metastasis, contralateral breast cancer, and intercurrent death. Competing risk regression was used to identify risk factors for LR. For patients with isolated LR after BCT, distant metastasis-free survival (DMFS) and overall survival (OS) were calculated from the date of pathologic confirmation of LR to the event of interest, using the Kaplan-Meier method. Univariate analysis was conducted using the log-rank test and multivariate analysis was conducted using Cox proportional hazard analysis. A two-sided $p$ value less than 0.05 was considered statistically significant. All statistical analyses were performed with $\mathrm{R}$ software ver. 3.3.3.

\section{Declarations}

\section{Acknowledgements}

This work was supported by Konyang University Myunggok Research Fund of 2017-01.

\section{Funding}

No funding was received.

\section{Availability of data and materials}

The datasets used and/or analyzed during the current study are available from the corresponding author on reasonable request.

\section{Authors' contributions}

S-WK and MC designed the study. YTh, OKN, YJ, DSY and MC collected the data. DVN, S-WK and MC analyzed the data. DVN and S-WK drafted the manuscript. MC supervised and revised the manuscript. All authors read and approved the final manuscript.

\section{Competing interest}

The authors have stated that they have no conflicts of interest.

\section{References}

1. Elkhuizen, P. H. et al. Local recurrence after breast-conserving therapy for invasive breast cancer: high incidence in young patients and association with poor survival. Int J Radiat Oncol Biol Phys. 40, 
859-867 (1998).

2. Vrieling, C. et al. Can patient-, treatment- and pathology-related characteristics explain the high local recurrence rate following breast-conserving therapy in young patients? Eur J Cancer. 39, 932-944 (2003).

3. Anders, C. K. et al. Young age at diagnosis correlates with worse prognosis and defines a subset of breast cancers with shared patterns of gene expression. J Clin Oncol. 26, 3324-3330 https://doi.org/10.1200/JC0.2007.14.2471 (2008).

4. Fisher, B. et al. Twenty-year follow-up of a randomized trial comparing total mastectomy, lumpectomy, and lumpectomy plus irradiation for the treatment of invasive breast cancer. $N$ Engl $J$ Med. 347, 1233-1241 https://doi.org/10.1056/NEJMoa022152 (2002).

5. Veronesi, U. et al. Twenty-year follow-up of a randomized study comparing breast-conserving surgery with radical mastectomy for early breast cancer. N Engl J Med. 347, 1227-1232 https://doi.org/10.1056/NEJMoa020989 (2002).

6. Kroman, N. et al. Effect of breast-conserving therapy versus radical mastectomy on prognosis for young women with breast carcinoma. Cancer. 100, 688-693 https://doi.org/10.1002/cncr.20022 (2004).

7. Coulombe, G. et al. Is mastectomy superior to breast-conserving treatment for young women? Int $J$ Radiat Oncol Biol Phys. 67, 1282-1290 https://doi.org/10.1016/j.jirobp.2006.11.032 (2007).

8. Beadle, B. M. et al. Ten-year recurrence rates in young women with breast cancer by locoregional treatment approach. Int J Radiat Oncol Biol Phys. 73, 734-744 https://doi.org/10.1016/j.jijobp.2008.04.078 (2009).

9. Bantema-Joppe, E. J. et al. Early-stage young breast cancer patients: impact of local treatment on survival. Int J Radiat Oncol Biol Phys. 81, e553-559 https://doi.org/10.1016/j.ijrobp.2011.02.060 (2011).

10. van der Sangen, M. J. et al. Are breast conservation and mastectomy equally effective in the treatment of young women with early breast cancer? Long-term results of a population-based cohort of 1,451 patients aged. Breast Cancer Res Treat. 127, 207-215 https://doi.org/10.1007/s10549-0101110-x (2011).

11. Mahmood, U. et al. Similar survival with breast conservation therapy or mastectomy in the management of young women with early-stage breast cancer. Int J Radiat Oncol Biol Phys. 83, 1387-1393 https://doi.org/10.1016/j.ijrobp.2011.10.075 (2012).

12. Bantema-Joppe, E. J. et al. Impact of primary local treatment on the development of distant metastases or death through locoregional recurrence in young breast cancer patients. Breast Cancer Res Treat. 140, 577-585 https://doi.org/10.1007/s10549-013-2650-7 (2013).

13. Jeon, Y. W. et al. Impact of local surgical treatment on survival in young women with T1 breast cancer: long-term results of a population-based cohort. Breast Cancer Res Treat. 138, 475-484 https://doi.org/10.1007/s10549-013-2456-7 (2013). 
14. Cao, J. Q. et al. Should women younger than 40 years of age with invasive breast cancer have a mastectomy? 15-year outcomes in a population-based cohort. Int J Radiat Oncol Biol Phys. 90, 509517 https://doi.org/10.1016/j.ijrobp.2014.06.041 (2014).

15. Xie, Z. et al. Breast-conserving therapy: a viable option for young women with early breast cancerevidence from a prospective study. Ann Surg Oncol. 21, 2188-2196 https://doi.org/10.1245/s10434014-3620-y (2014).

16. Frandsen, J. et al. In the Modern Treatment Era, Is Breast Conservation Equivalent to Mastectomy in Women Younger Than 40 Years of Age? A Multi-Institution Study. Int J Radiat Oncol Biol Phys. 93, 1096-1103 https://doi.org/10.1016/j.ijrobp.2015.08.044 (2015).

17. Ye, J. C., Yan, W., Christos, P. J., Nori, D. \& Ravi, A. Equivalent Survival With Mastectomy or Breastconserving Surgery Plus Radiation in Young Women Aged $<40$ Years With Early-Stage Breast Cancer: A National Registry-based Stage-by-Stage Comparison. Clin Breast Cancer. 15, 390-397 https://doi.org/10.1016/j.clbc.2015.03.012 (2015).

18. Plichta, J. K. et al. Factors Associated with Recurrence Rates and Long-Term Survival in Women Diagnosed with Breast Cancer Ages 40 and Younger. Ann Surg Oncol. 23, 3212-3220 https://doi.org/10.1245/s10434-016-5404-z (2016).

19. Quan, M. L. et al. The effect of surgery type on survival and recurrence in very young women with breast cancer. J Surg Oncol. 115, 122-130 https://doi.org/10.1002/jso.24489 (2017).

20. Maishman, T. et al. Local Recurrence and Breast Oncological Surgery in Young Women With Breast Cancer: The POSH Observational Cohort Study. Ann Surg. 266, 165-172 https://doi.org/10.1097/SLA.0000000000001930 (2017).

21. van Laar, C. et al. Local recurrence following breast-conserving treatment in women aged 40 years or younger: trends in risk and the impact on prognosis in a population-based cohort of 1143 patients. Eur J Cancer. 49, 3093-3101 https://doi.org/10.1016/j.ejca.2013.05.030 (2013).

22. Botteri, E. et al. Improved prognosis of young patients with breast cancer undergoing breastconserving surgery. Br J Surg. https://doi.org/10.1002/bjs.10658 (2017).

23. Cardoso, F. et al. The European Society of Breast Cancer Specialists recommendations for the management of young women with breast cancer. Eur J Cancer. 48, 3355-3377 https://doi.org/10.1016/j.ejca.2012.10.004 (2012).

24. Partridge, A. H. et al. First international consensus guidelines for breast cancer in young women (BCY1). Breast. 23, 209-220 https://doi.org/10.1016/j.breast.2014.03.011 (2014).

25. Kurian, A. W. et al. Use of and mortality after bilateral mastectomy compared with other surgical treatments for breast cancer in California, 1998-2011. JAMA. 312, 902-914 https://doi.org/10.1001/jama.2014.10707 (2014).

26. Pesce, C. E., Liederbach, E., Czechura, T., Winchester, D. J. \& Yao, K. Changing surgical trends in young patients with early stage breast cancer, 2003 to 2010: a report from the National Cancer Data Base. $J$ Am Coll Surg. 219, 19-28 https://doi.org/10.1016/j.jamcollsurg.2014.03.043 (2014). 
27. Anderson, S. J. et al. Prognosis after ipsilateral breast tumor recurrence and locoregional recurrences in patients treated by breast-conserving therapy in five National Surgical Adjuvant Breast and Bowel Project protocols of node-negative breast cancer. J Clin Oncol. 27, 2466-2473 https://doi.org/10.1200/JC0.2008.19.8424 (2009).

\section{Figures}

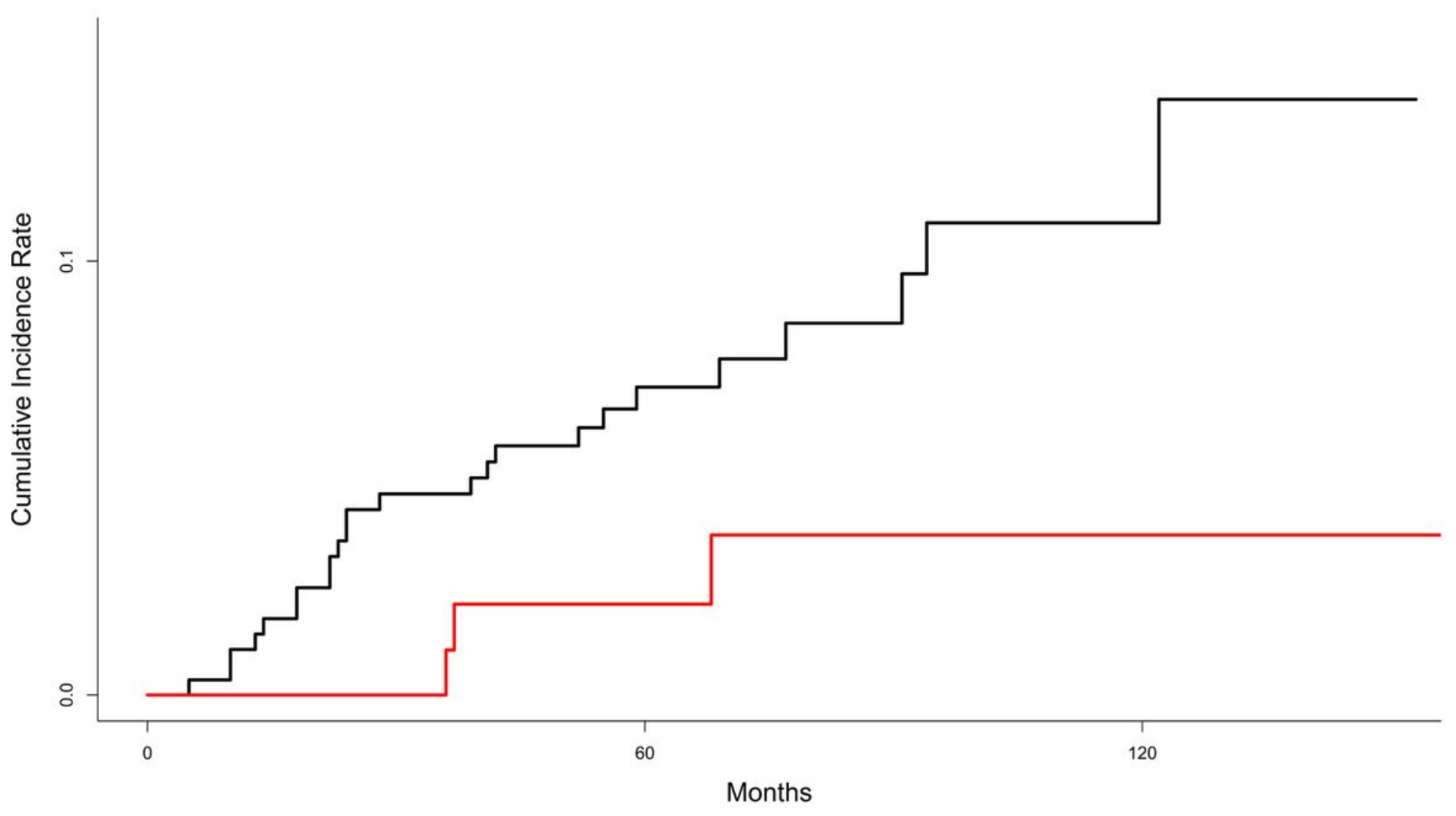

Figure 1

Graph of cumulative incidence of local recurrence between breast conservation therapy (black line) and mastectomy alone (red line) 


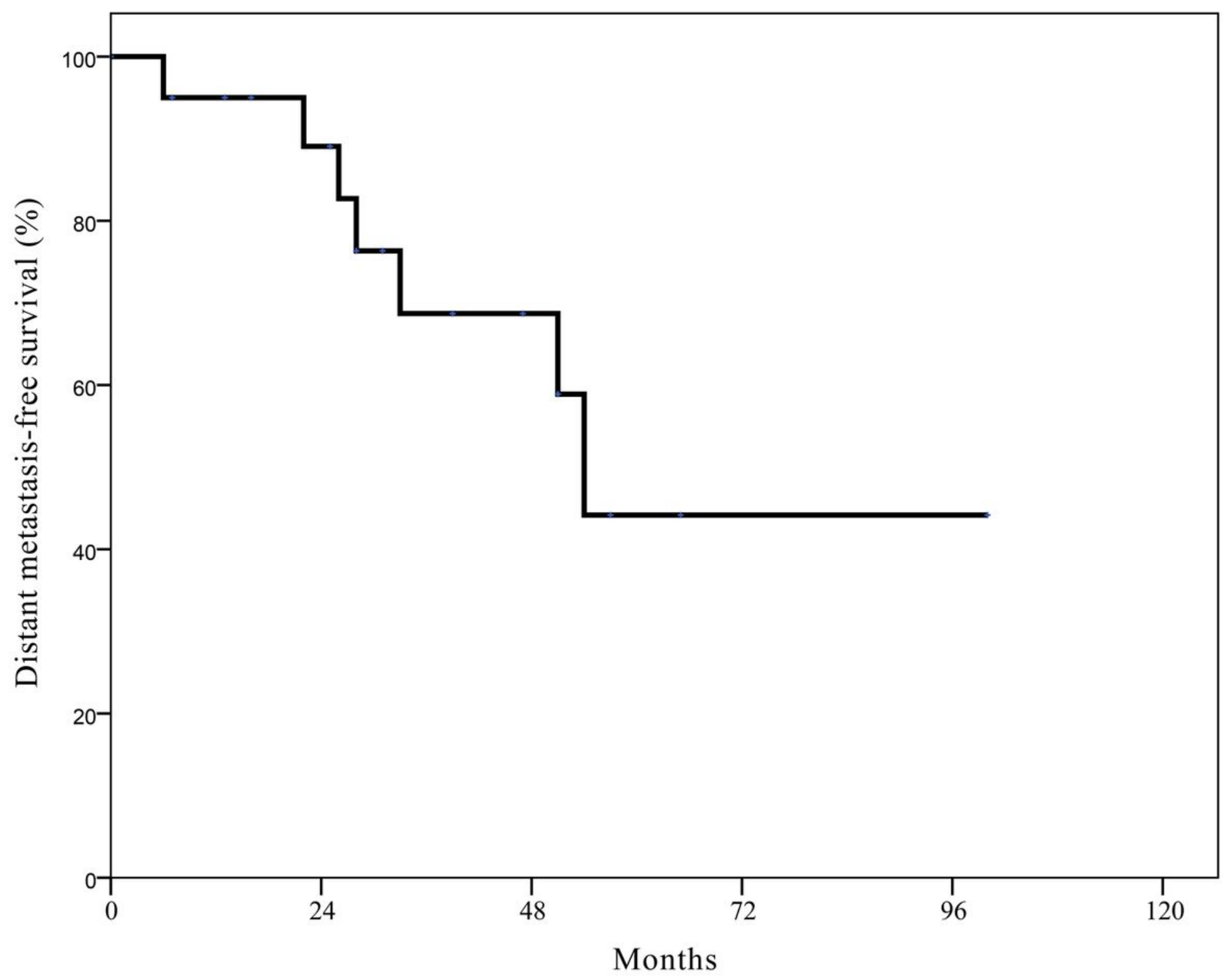

Figure 2

Kaplan-Meier curve of distant metastasis-free survival in patients with isolated local recurrence after breast conservation therapy. 


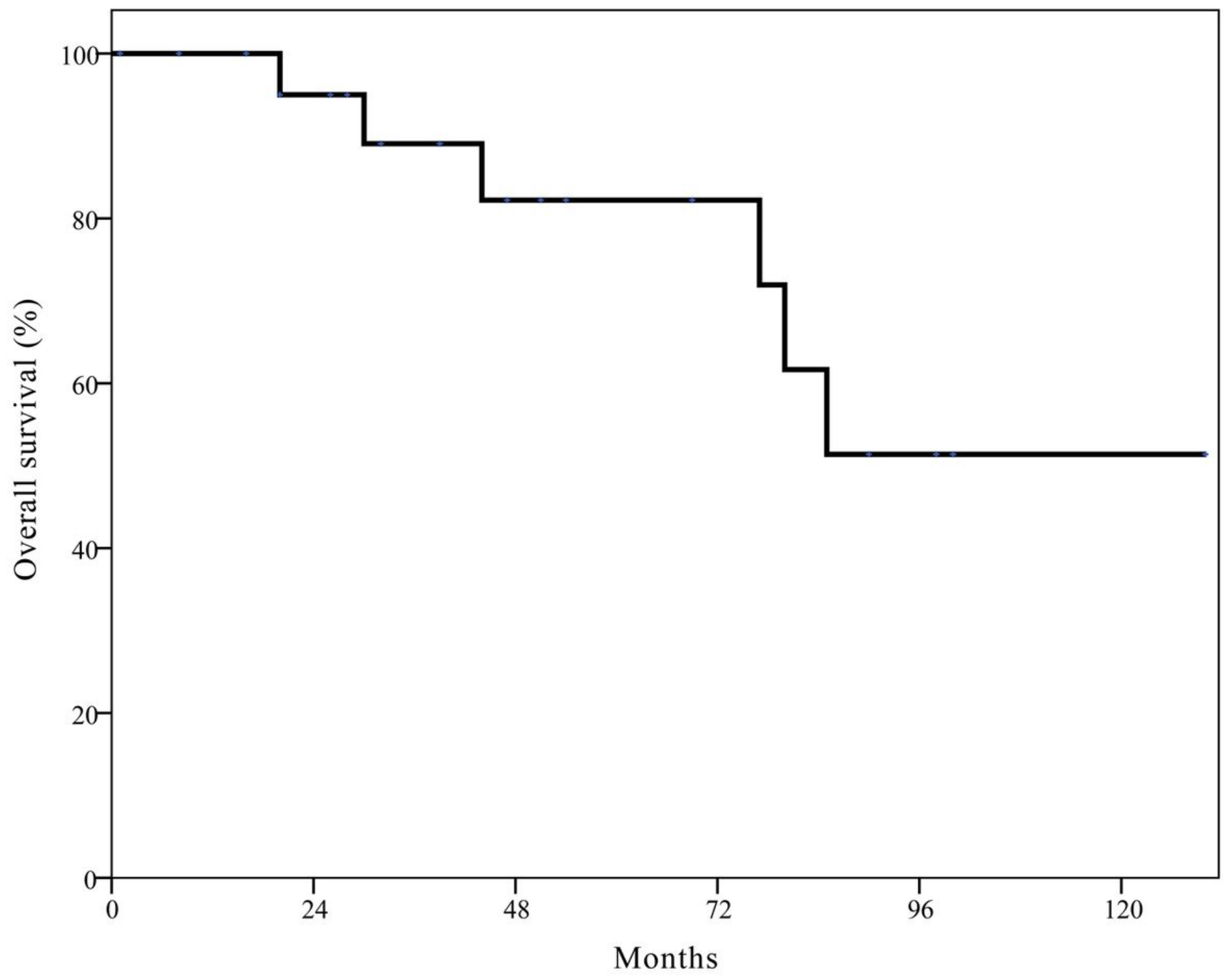

Figure 3

Kaplan-Meier curve of overall survival in patients with isolated local recurrence after breast conservation therapy. 